2. The frequency of the line at 2907.4 is fairly well represented by the equation

$$
\begin{aligned}
& v=\left(2, p_{2}, \pi_{2}\right)-\mathrm{B}(\mathrm{I})-\left(5, p_{2}, \pi_{2}\right), \\
& v=34,451.4 \text { calculated, } \\
& v=34,395.99 \text { observed. }
\end{aligned}
$$

The above symbols have the following values as given in Seriengesetze der Linienspektra by Dr. B. Dunz.

$$
\begin{aligned}
\left(2, p_{2}, \pi_{2}\right) & =48,279 \cdot 5 \mathrm{I} \\
\mathrm{B}(\mathrm{I}) & =8,882 . \mathrm{I} 9 \\
\left(3, p_{2}, \pi_{2}\right) & =\mathrm{I} 5,33 \mathrm{I} .00 \\
\left(5, p_{2}, \pi_{2}\right) & =4,945.96 .
\end{aligned}
$$

While the agreement between calculated and observed values is not as close as might be desired the similarity in meaning of the two equations is suggestive.

University of Chicago.

\title{
The Aspherical Nucleus Theory Applied to the Principal Series of Helium.
}

By Ludwik Silberstein.

THE quantum theory of spectrum emission combined with the assumption of a non-spherical, axially symmetrical nucleus, is applied to Fowler's principal series of helium, corresponding to the fixed total quantum number $n^{\prime}=3$ and the variable ones $n=4,5$, etc. The theoretical 'main' components, i.e., those corresponding to the passage of the electron from a circular-equatorial to a circular-equatorial orbit, are correlated with the strongest components observed by Paschen in the first five groups or members of the series and with the centres of the next two members observed (but not split) originally by Fowler. The two constants appearing in the general formula, the modified Rydberg constant $N$ and the coëfficient of asphericity $\sigma$ are thus determined. The differences between the calculated and the observed wave-lengths are all within the error limits. The finally adopted values are $\sigma=9.334 \cdot 1 \mathrm{O}^{-4}$ and $N=$ I09723.22. The latter, combined with a previously obtained value of $N$ for hydrogen, gives for the ratio of the mass of the hydrogen atom to that of the electron the value I8I7. The fine-structure depending on $\sigma$, through the product $N \sigma$, is then investigated and discussed numerically in the case of the first two members of the series, the groups 4686 and 3203. The distribution of the theoretical components shows, especially in the case of the first group, some similarity to Paschen's observations, although there are superabundant theoretical components.

EASTMAN KODAK COMPANy. 